\title{
Mitochondrial dysfunction induces the invasive phenotype, and cell migration and invasion, through the induction of AKT and AMPK pathways in lung cancer cells
}

\author{
SI-YOON HAN ${ }^{1,2^{*}}$, YUN-JEONG JEONG ${ }^{1,2^{*}}$, YONGSOO CHOI ${ }^{3,4}$, SOON-KYUNG HWANG ${ }^{1,2}$, \\ YOUNG-SEUK BAE ${ }^{5}$ and YOUNG-CHAE CHANG $^{1,2}$
}

${ }^{1}$ Department of Cell Biology; ${ }^{2}$ Research Institute of Biomedical Engineering, Catholic University of Daegu School of Medicine, Daegu 42472; ${ }^{3}$ Systems Biotechnology Research Center, Korea Institute of Science and Technology (KIST), Gangneung, Gangwon 25451; ${ }^{4}$ Department of Biological Chemistry, University of Science and Technology (UST), Daejeon 34113; ${ }^{5}$ School of Life Sciences, BK21 Plus KNU Creative BioResearch Group, College of Natural Sciences, Kyungpook National University, Daegu 41566, Republic of Korea

Received November 17, 2017; Accepted May 8, 2018

DOI: $10.3892 /$ ijmm.2018.3733

\begin{abstract}
Mitochondria are well known for their important roles in oxidative phosphorylation, amino acid metabolism, fatty acid oxidation and ion homeostasis. Although the effects of mitochondrial dysfunction on tumorigenesis in various cancer cells have been reported, the correlation between mitochondrial dysfunction and epithelial-to-mesenchymal transition (EMT) in lung cancer development and metastasis has not been well elucidated. In the present study, the effects of mitochondrial dysfunction on EMT and migration in lung cancer cells were investigated using inhibitors of mitochondrial respiration, oligomycin $\mathrm{A}$ and antimycin $\mathrm{A}$. Oligomycin $\mathrm{A}$ and antimycin A induced distinct mesenchymal-like morphological features in H23, H1793 and A549 lung cancer cells. In addition, they decreased the expression levels of the epithelial marker protein E-cadherin, but increased the expression levels of the mesenchymal marker proteins Vimentin, Snail and Slug. The results of immunofluorescence staining indicated that oligomycin A and antimycin A downregulated cortical E-cadherin expression and upregulated the expression of Vimentin. In addition, oligomycin A and antimycin A increased the migration and invasion of A549 lung cancer cells, and promoted the expression levels of phosphorylated (p)-protein kinase B (AKT) and p-AMP-activated protein
\end{abstract}

Correspondence to: Dr Young-Chae Chang, Department of Cell Biology, Catholic University of Daegu School of Medicine, 3056-6 Daemyung-4-Dong, Nam-gu, Daegu 42472, Republic of Korea

E-mail: ycchang@cu.ac.kr

*Contributed equally

Keywords: mitochondrial respiration, epithelial-mesenchymal transition, migration, invasion, lung cancer kinase (AMPK). Notably, the production of reactive oxygen species by oligomycin A and antimycin A did not affect the expression of EMT protein markers. Conversely, treatment with the AKT inhibitor wortmannin and the AMPK inhibitor Compound $\mathrm{C}$ upregulated E-cadherin and downregulated Vimentin expression. These results suggested that oligomycin $A$ and antimycin A may induce migration and invasion of lung cancer cells by inducing EMT via the upregulation of $\mathrm{p}-\mathrm{AKT}$ and $\mathrm{p}-\mathrm{AMPK}$ expression.

\section{Introduction}

Epithelial-to-mesenchymal transition (EMT) is a necessary condition associated with numerous cancer developmental processes, including tumor invasion and metastasis, in various types of epithelial cancer $(1,2)$. When EMT occurs, epithelial cells lose their cell adhesion and apical-basal polarity. In addition, epithelial cell-matrix interactions are altered due to reorganization of the actin cytoskeleton, and cells gain a mesenchymal phenotype with high mobility (3). During the EMT process, EMT is frequently characterized by the reduced expression of E-cadherin, N-cadherin and other epithelial markers, and by the increased expression of Vimentin, Snail and other mesenchymal markers (4). Mesenchymal cells infiltrate blood vessels and lymph nodes, and may then migrate to other tissues, resulting in tumor formation (5). Numerous studies have demonstrated that activity of the phosphoinositide 3-kinase (PI3K)/protein kinase B (AKT) pathway serves an important role in upregulating cell proliferation, migration and EMT $(6,7)$. In addition to this pathway, the EMT process can promote the activity of AMP-activated protein kinase (AMPK), which is an important regulator of glucose uptake and energy balance (8). A previous study demonstrated that activation of AMPK could induce migration by increasing the expression levels of microRNA-451 in glioblastoma (9). The oxidative phosphorylation (OXPHOS) process through the mitochondrial electron transport system in cells is influenced by the intracellular energy metabolic activity of AMPK. 
Mitochondria serve a central role in the production of cellular energy and in metabolism through the OXPHOS process of the electron transport system via respiration (10). Mitochondrial dysfunction has been reported to be associated with the development of various types of human cancer, and it occurs through the production of reactive oxygen species (ROS), which are substrates of oxidative phosphorylation (11), through the reduction and the activation of mitochondria respiration chain complex. In addition, the high mutation rate of mitochondrial (mt)DNA and nuclear gene-coded electron transport chain proteins are known to influence mitochondrial dysfunction (12), and have been reported to regulate apoptosis or programmed cell death (13). Oligomycin A is an inhibitor of ATP synthase, and antimycin A is a specific inhibitor of mitochondrial electron transport complex I, which previously led to an increase in gastric cancer progression via the promotion of ROS (14). In addition, oligomycin A treatment increased cytosolic $\mathrm{Ca}^{2+}$ and ROS in HepG2 cells, and stimulated migration in SK-Hep-1 cells (15).

Although inhibitors of mitochondrial respiration, including oligomycin A and antimycin A, have been reported to have an effect on metastasis in various types of human cancer cells, the molecular mechanism underlying mitochondrial respiration inhibitor-mediated EMT in lung cancer cells remains to be elucidated. Therefore, the present study aimed to determine the effects of mitochondrial respiration inhibitors oligomycin A and antimycin A on EMT, and on cell migration and invasion, in the A549 human lung cancer cell line. The results demonstrated that treatment with mitochondrial respiration inhibitors may induce the invasive phenotype, as well as cell migration and invasion. Furthermore, treatment with mitochondrial respiration inhibitors increased the protein expression levels of mesenchymal markers via induction of the AKT and AMPK signaling pathways.

\section{Materials and methods}

Cells and materials. A549, H23 and H1793 lung carcinoma cells were obtained from the American Type Culture Collection (Manassas, VA, USA). Cells were cultured in RPMI 1640 (Gibco; Thermo Fisher Scientific, Inc., Waltham, MA, USA) supplemented with $10 \%$ fetal bovine serum (HyClone; GE Healthcare Life Sciences, Logan, UT, USA) in an atmosphere containing $5 \% \mathrm{CO}_{2}$ at $37^{\circ} \mathrm{C}$. Oligomycin $\mathrm{A}$ and antimycin A were obtained from Sigma-Aldrich; Merck KGaA (Darmstadt, Germany). All chemicals, including $\mathrm{N}$-acetyl-L-cysteine (NAC; ROS scavenger), wortmannin (PI3K inhibitor) and Compound $\mathrm{C}$ (AMPK inhibitor) were obtained from Sigma-Aldrich; Merck KGaA, unless otherwise indicated. Densitometric analysis was performed on scanned western blot and reverse transcription-polymerase chain reaction (RT-PCR) images using ImageJ software (version 2018; National Institutes of Health, Bethesda, MD, USA).

Cell viability assay. Human non-small cell lung cancer cell lines were seeded in 96 -well plates at $2 \times 10^{4}$ cells/well in RPMI 1640 media, and were allowed to adhere to the surface for $24 \mathrm{~h}$. Subsequently, fresh media containing various concentrations of oligomycin A and antimycin A were added to the plates, and cells were incubated for $24 \mathrm{~h}$. Subsequently, $500 \mu \mathrm{g} / \mathrm{ml}$
MTT (Roche Diagnostics, Indianapolis, IN, USA) was added to each well. The amount of formazan deposits were dissolved in $100 \mu 1$ dimethyl sulfoxide and absorbance was measured at $540 \mathrm{~nm}$ using a plate reader (VersaMax ${ }^{\mathrm{TM}}$ Microplate Reader; Molecular Devices, LLC, Sunnyvale, CA, USA), following a $4 \mathrm{~h}$ incubation with MTT in an atmosphere containing $5 \% \mathrm{CO}_{2}$ at $37^{\circ} \mathrm{C}$.

Morphological analysis. Cells were cultured in a $60 \mathrm{~mm}$ dish and were cultured until they reached $60 \%$ confluence. Subsequently, the media were replaced with fresh RPMI 1640 media, and the cells were incubated with $1 \mu \mathrm{g} / \mathrm{ml}$ oligomycin A or antimycin A for $24 \mathrm{~h}$ in an atmosphere containing $5 \% \mathrm{CO}_{2}$ at $37^{\circ} \mathrm{C}$. Finally, images of the cells were captured using amicroscope (Nikon Eclipse TS100, Nikon Corporation, Tokyo, Japan).

Western blot analysis. Cells were seeded in a $60-\mathrm{mm}$ dish at $1 \times 10^{6}$ cells/well in RPMI-1640 media, and were allowed to adhere to the surface for $24 \mathrm{~h}$. Subsequently, fresh media containing various concentrations of oligomycin A and antimycin A were added to the plates, and cells were incubated for $24 \mathrm{~h}$. Cell lysis, SDS-PAGE, transfer to a nitrocellulose membrane (EMD Millipore, Billerica, MA, USA) and immunoblotting were performed as previously described (16). Briefly, cells were suspended in $0.1 \mathrm{ml}$ lysis buffer containing 10 mM HEPES (pH 7.9), 10 mM KCl, 0.1 mM EDTA, $0.1 \mathrm{mM}$ EGTA, $1 \mathrm{mM}$ DTT, $0.5 \mathrm{mM}$ phenylmethlysulfonyl fluoride, $2.0 \mu \mathrm{g} / \mathrm{ml}$ leupeptin and $2.0 \mu \mathrm{g} / \mathrm{ml}$ aprotinin at $4^{\circ} \mathrm{C}$ for $30 \mathrm{~min}$. Subsequently, cells were centrifuged at $14,645 \mathrm{x}$ g for $10 \mathrm{~min}$. Protein concentration was measured using the Bicinchoninic Acid (BCA) Assay (Pierce ${ }^{\mathrm{TM}}$ BCA Protein Assay kit; Thermo Fisher Scientific, Inc.) Total proteins $(30 \mu \mathrm{g})$ were separated by $8-15 \%$ SDS-PAGE and were transferred to nitrocellulose membranes. The membranes were then blocked in $5 \%$ skim milk in Tris-buffered saline containing $0.1 \%$ Tween-20 (TBS-T) for $1 \mathrm{~h}$ at room temperature. The membranes were then incubated with primary antibodies at $4^{\circ} \mathrm{C}$ overnight, washed three times with TBS-T, and incubated with goat anti-mouse immunoglobulin G (IgG; \#31430) or goat anti-rabbit IgG (\#65-6120) secondary antibodies (Thermo Fisher Scientific, Inc.) at a dilution of 1:2,000 for $1 \mathrm{~h}$ at room temperature, before being washed a further three times with TBS-T. Signals were detected using enhanced chemiluminescence (GE Healthcare, Chicago, IL, USA) and ChemiDOC ${ }^{\mathrm{TM}}$ XRS (Bio-Rad Laboratories, Inc., Hercules, CA, USA). The primary antibodies at a dilution of 1:1,000 used in the present study were: E-cadherin (BD610182, BD Biosciences, Franklin Lakes, NJ, USA); Snail (\#3879), Slug (\#9585), phosphorylated (p)-AMPKa (\#2531), p-acetyl-CoA carboxylase (ACC; \#3661) and p-AKT (\#4060) (all from Cell Signaling Technology, Inc., Danvers, MA, USA); Vimentin (sc-6260) and $\beta$-actin (sc-47778) (Santa Cruz Biotechnology, Dallas, TX, USA).

$R T$-PCR . Cells were seeded in a $60 \mathrm{~mm}$ dish at $1 \times 10^{6}$ cells/well in RPMI 1640 media, and were allowed to adhere to the surface for $24 \mathrm{~h}$. Subsequently, fresh media containing $1 \mu \mathrm{g} / \mathrm{ml}$ oligomycin A and antimycin A were added to the plates, and cells were incubated for $24 \mathrm{~h}$. Total RNA was extracted using TRIzol ${ }^{\circledR}$ (Invitrogen; Thermo Fisher Scientific, Inc.), 
according to the manufacturer's protocol. For RT-PCR, cDNA was synthesized from $1 \mu \mathrm{g}$ total RNA using AccuPower ${ }^{\circledR}$ RT PreMix (Bioneer Corporation, Daejeon, Korea), according to the manufacturer's protocol. cDNA was amplified by PCR (AccuPower ${ }^{\circledR}$ RT-PCR PreMix; Bioneer Corporation) with the following primers: Snail, sense 5'-CAGCGAGCTGCAGGA CTCTA-3', antisense 5'-GTGGGATGGCTGCCAGC-3'; Slug, sense 5'-TGTGTGGACTACCGCTGC-3', antisense 5'-TCC GGAAAGAGGAGAGAGG-3'; and $\beta$-actin, sense 5'-CAA GAGATGGCCACGGCTGCT-3' and antisense 5'-TCCTTC TGCATCCTGTCGGCA-3'. PCR products were analyzed by $1.5 \%$ agarose gel electrophoresis and visualized using ethidium bromide (E1510; Sigma-Aldrich; Merck KGaA) and ChemiDOC $^{\text {TM }}$ XRS (Bio-Rad Laboratories, Inc.).

Transwell invasion assay. Matrigel-coated filter inserts (pore size, $8 \mu \mathrm{m}$; SPLInsert ${ }^{\mathrm{TM}}$ Hanging) that fit into 24-well migration chambers were obtained from SPL Life Sciences (Pocheon, Korea). Cells were plated at $2 \times 10^{4}$ cells/well in the upper chamber. The lower chamber was filled with culture media containing $1 \mu \mathrm{g} / \mathrm{ml}$ oligomycin A or antimycin A. Cells were incubated in the chamber for $24 \mathrm{~h}$ at $37^{\circ} \mathrm{C}$; subsequently, cells that invaded across the membrane surface were fixed with $100 \%$ methanol for $1 \mathrm{~min}$ and stained with $0.5 \%$ crystal violet for $10 \mathrm{~min}$ at room temperature. The cells that passed through the Matrigel-coated inserts and were located on the underside of the filter were counted. Random fields were counted by light microscopy (x200 magnification).

Wound-healing assay. The wound-healing assay was performed according to a previously described procedure, with minor modifications (17). Briefly, cells were seeded in 6-well plates and were incubated until they reached $80 \%$ confluence. Cell monolayers were then scratched with a 200- $\mu$ l pipette tip to create a wound, and cells were washed twice with serum-free culture media to remove floating cells. Media were then replaced with fresh serum-free media and cells were subjected to treatment with $1 \mu \mathrm{g} / \mathrm{ml}$ oligomycin A or antimycin A for $24 \mathrm{~h}$ at $37^{\circ} \mathrm{C}$; images of the cells were captured after $24 \mathrm{~h}$ using a microscope (Nikon Eclipse TS100; Nikon Corporation).

Immunofluorescence. For immunofluorescence, cells were cultured on cover slips at $50 \%$ confluence, and were cultured with $1 \mu \mathrm{g} / \mathrm{ml}$ oligomycin A or antimycin A for $24 \mathrm{~h}$ at $37^{\circ} \mathrm{C}$. Cells on cover slips were washed with PBS and were fixed in $100 \%$ methanol for $1 \mathrm{~min}$ and permeabilized with $0.2 \%$ Triton X-100 in PBS for 5 min. Cells were then blocked with 10\% normal goat serum (\#31872; Invitrogen; Thermo Fisher Scientific, Inc.) in PBS for $1 \mathrm{~h}$ at room temperature and were then incubated with primary antibodies against E-cadherin (\#3195; Cell Signaling Technology, Inc.) and Vimentin (sc-6260; Santa Cruz Biotechnology, Inc.). The cells were washed three times with PBS and were incubated with Alexa Fluor ${ }^{\circledR}$ 488-conjugated anti-mouse IgG (\#R37120; Invitrogen; Thermo Fisher Scientific, Inc.) and Flamma 648-conjugated anti-rabbit IgG (RSA1261a; BioActs, Incheon, Korea) secondary antibodies diluted 1:200 in blocking buffer. Fluorescent signals were visualized by confocal microscopy (Leica Microsystems GmbH, Wetzlar, Germany).
Statistical analysis. All in vitro results were derived from at least three independent experiments performed in triplicate. Data were analyzed using GraphPad Prism 5 software (GraphPad Software, Inc., La Jolla, CA, USA). The significance of differences between experimental and control groups was determined using one-way analysis of variance followed by Newman-Keuls multi-comparison test (Prism 5. GraphPad Software, Inc., CA, USA). P $<0.05$ was considered to indicate a statistically significant difference.

\section{Results}

Inhibitors of mitochondrial respiration induce mesenchymal morphology in lung cancer cells. Prior to investigating the effects of oligomycin A and antimycin A on EMT, the cytotoxic effects of oligomycin A and antimycin A were examined using the MTT assay. The results demonstrated that viability was $>80 \%$ in A549, $\mathrm{H} 23$ and $\mathrm{H} 1793$ cells following treatment with oligomycin $\mathrm{A}$ and antimycin $\mathrm{A}$ at doses $<1 \mu \mathrm{g} / \mathrm{ml}$. Cytotoxic effects of oligomycin A and antimycin A were observed following treatment at $2 \mu \mathrm{g} / \mathrm{ml}$ in A549 cells (Fig. 1A). The present study aimed to determine whether the inhibitors of mitochondrial respiration, oligomycin A and antimycin A, may induce EMT in human epithelial lung cancer cell lines, including A549, H23 and H1793. Cell morphology was observed by microscopy; the results demonstrated that untreated lung cancer cells (control) exhibited cobblestone-like cell morphology and cluster formation, which are characteristic of epithelial cells (Fig. 1B). Notably, following treatment with $1 \mu \mathrm{g} / \mathrm{ml}$ oligomycin A or antimycin A for $24 \mathrm{~h}$, the cells exhibited mesenchymal-like morphological features, including a spindle shape and reduced intercellular contacts. The regulatory effects of mitochondrial respiration inhibitors on the EMT-associated markers E-cadherin and Vimentin were determined in lung cancer cells using western blotting. As shown in Fig. 2A and B, the protein expression levels of E-cadherin were significantly decreased by treatment with oligomycin A and antimycin A at the indicated concentrations; however, oligomycin A and antimycin A markedly increased Vimentin protein expression. These results suggested that mitochondrial respiration inhibitors may promote EMT. In addition, oligomycin A and antimycin A enhanced the downregulation of E-cadherin and the upregulation of Vimentin in a time-dependent manner (Fig. 2C and D). Since mitochondrial respiration inhibitor-induced EMT was most prominent in A549 cells among the three types of epithelial lung cancer cells, subsequent experiments were performed on the A549 cell line.

Inhibitors of mitochondrial respiration induce EMT-associated transcriptional activation by increasing mesenchymal marker expression. To confirm whether the oligomyicin A- and antimycin A-induced mesenchymal-like morphology of lung cancer cells was closely associated with EMT, immunofluorescence staining was performed using E-cadherin and Vimentin antibodies. The results detected a decrease in E-cadherin expression and an increase in Vimentin expression upon oligomycin A $(1 \mu \mathrm{g} / \mathrm{ml})$ and antimycin A $(1 \mu \mathrm{g} / \mathrm{ml})$ treatment of A549 lung cancer cells (Fig. 3A). 
A
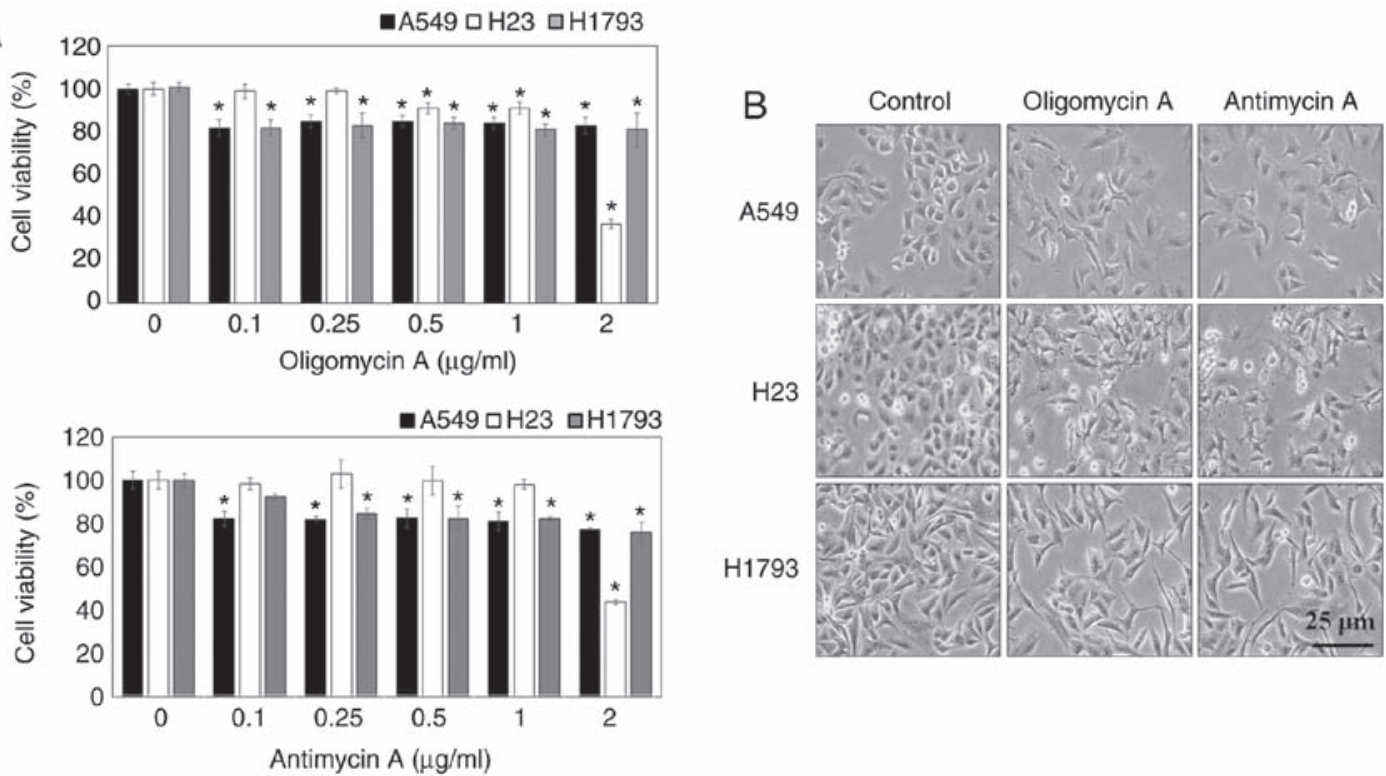

Figure 1. Inhibitors of mitochondrial respiration induce mesenchymal morphological alterations in lung cancer cells. (A) A549, H23 and H1793 cells were treated with the indicated concentrations of oligomycin A and antimycin A for $24 \mathrm{~h}$, after which cell viability was measured using the MTT assay. Data are presented as the means \pm standard error of three independent experiments. "P $<0.05$ vs. the control group. (B) A549, H23 and H1793 cells were treated with $1 \mu \mathrm{g} / \mathrm{ml}$ oligomycin A or antimycin A for $24 \mathrm{~h}$ and cell morphology was observed via microscopy.
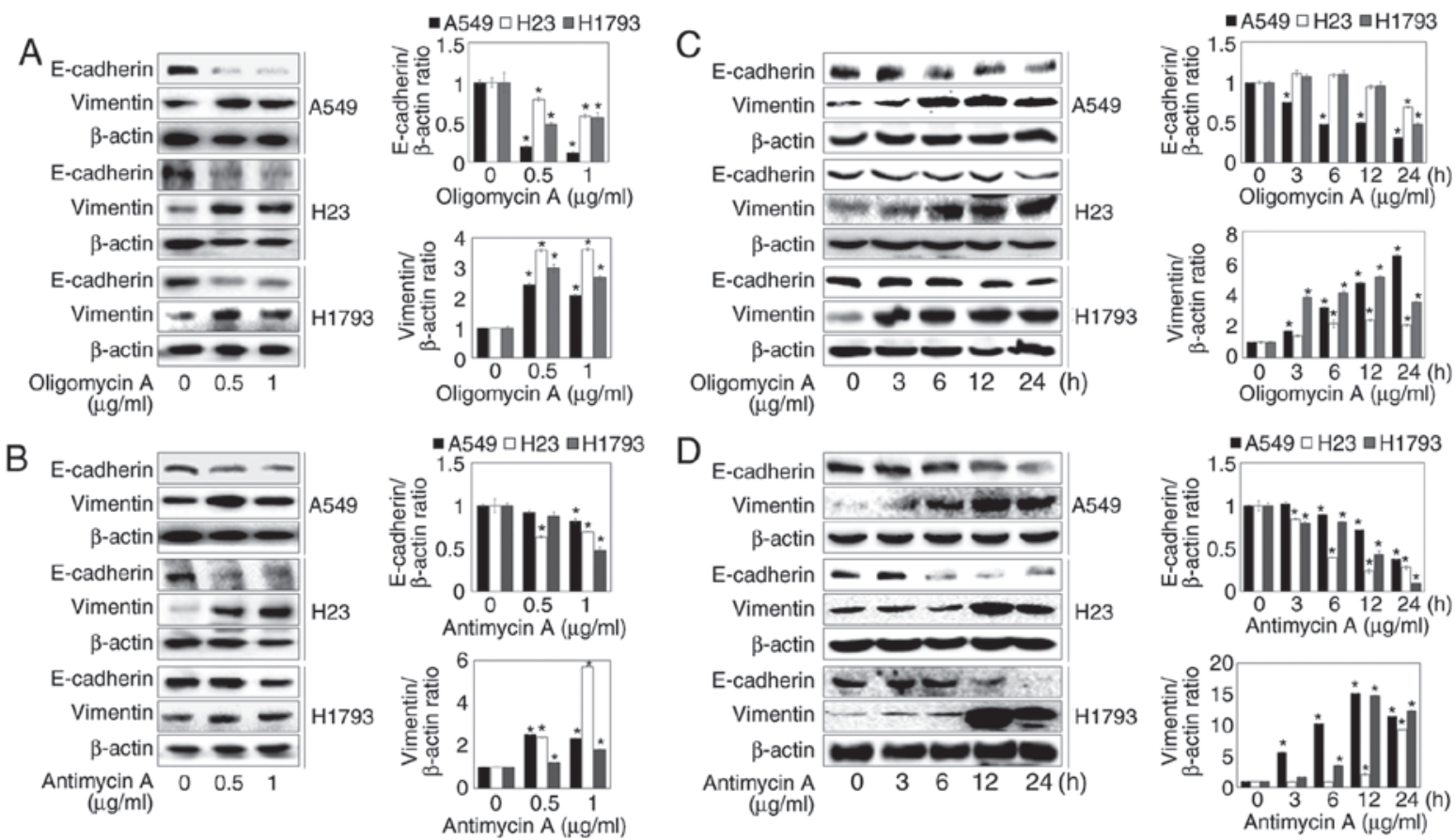

Figure 2. Inhibitors of mitochondrial respiration increase EMT protein marker expression in lung cancer cells. (A and B) A549, H23 and H1793 cells were treated with the indicated concentrations of oligomycin A and antimycin A for $24 \mathrm{~h}$. (C and D) Cells were treated with $1 \mu \mathrm{g} / \mathrm{ml}$ oligomycin A and antimycin A for the indicated durations, and E-cadherin and Vimentin protein expression levels were determined by western blot analysis. $\beta$-actin was used as a control. Densitometric analysis of each protein ratio was performed using ImageJ. Data are presented as the means \pm standard error of three independent experiments. ${ }^{*} \mathrm{P}<0.05$ vs. the control group.

Snail is a prominent inducer of EMT and strongly suppresses E-cadherin expression. Snail and Slug are zinc-finger transcriptional factors that downregulate E-cadherin expression and upregulate Vimentin expression by binding E-boxes (18). Therefore, in order to determine if the mitochondrial respiration inhibitors induce EMT by regulating transcription factors, the protein expression levels of Snail and Slug were detected. As shown in Fig. 3B and C, oligomycin A and antimycin A increased the protein expression levels of Snail and Slug. In addition, the mRNA expression levels of Snail and Slug were increased by oligomycin A and antimycin A (Fig. 3D), thus suggesting that mitochondrial respiration inhibitors may enhance the downregulation of E-cadherin and the upregulation of Vimentin by promoting Snail and Slug expression in A549 lung cancer cells. 


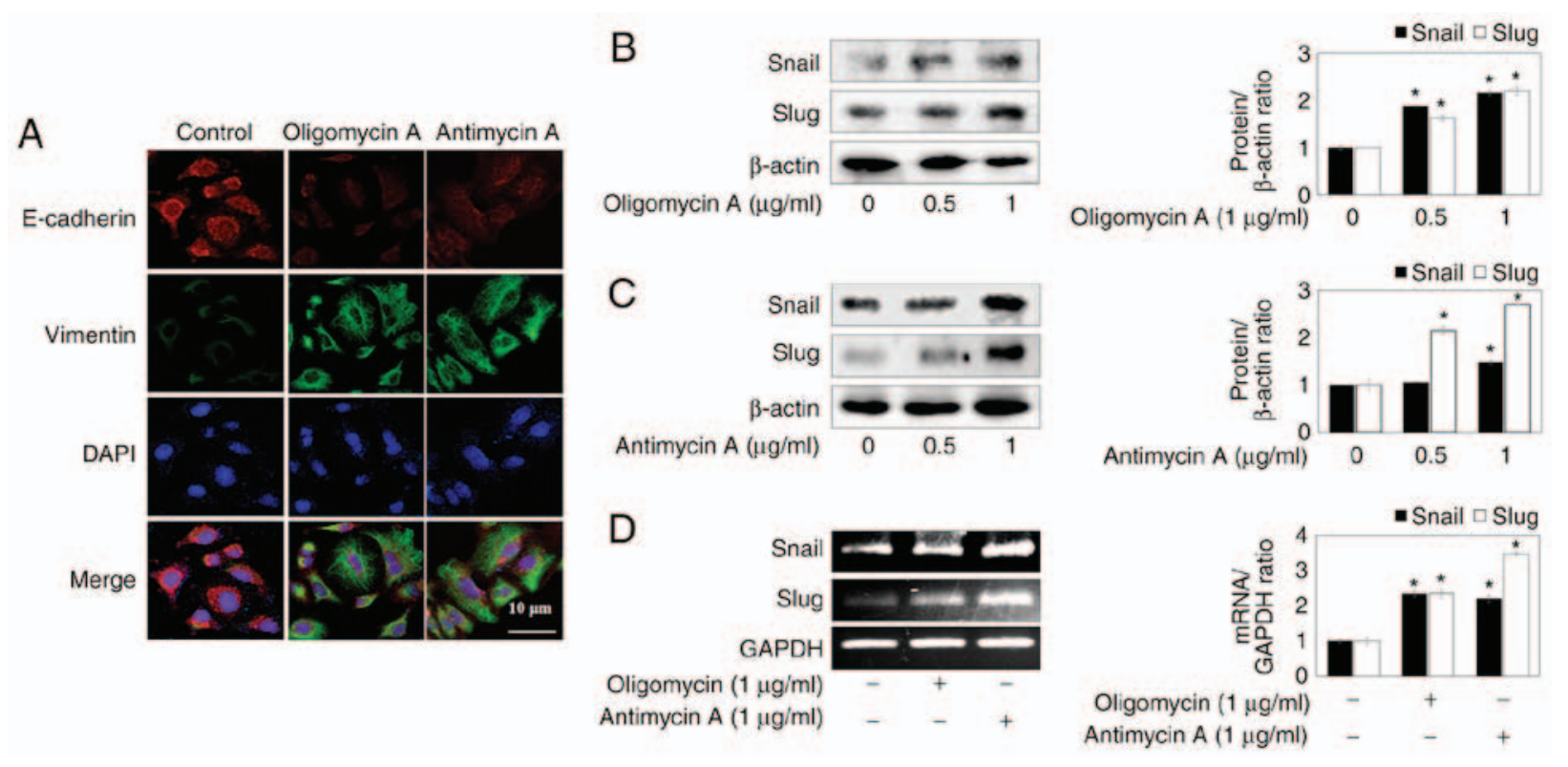

Figure 3. Inhibitors of mitochondrial respiration induce EMT in A549 cells. (A) E-cadherin and Vimentin expression were assessed in A549 cells after 24 h treatment with $1 \mu \mathrm{g} / \mathrm{ml}$ oligomycin A and antimycin A. E-cadherin and Vimentin were detected via immunofluorescence of formaldehyde-fixed cells with specific antibodies and a Alexa Fluor ${ }^{\circledR}$ 488-labeled secondary antibody (green, Vimentin) or Flamma ${ }^{\circledR}$ 648-labelled secondary antibody (red, E-cadherin). Nuclei were counterstained with DAPI. Magnification, x400. (B and C) Protein expression levels of the EMT transcription factors Snail and Slug were detected in A549 cells after $24 \mathrm{~h}$ treatment with the indicated concentrations of oligomycin A and antimycin A. (D) mRNA expression levels of Snail and Slug were detected in A549 cells after $24 \mathrm{~h}$ treatment with $1 \mu \mathrm{g} / \mathrm{ml}$ oligomycin A and antimycin A. Snail and Slug were detected by reverse transcription-polymerase chain reaction. $\beta$-actin and GAPDH were used as controls. Densitometric analysis of each protein and mRNA ratio was performed using ImageJ. Data are presented as the means \pm standard error of three independent experiments. ${ }^{*} \mathrm{P}<0.05$ vs. the control group.

Inhibitors of mitochondrial respiration increase cell migration and invasion of A549 lung cancer cells. EMT is an important process in epithelial-derived tumor malignancies, and is associated with cell adhesion. In addition, a partial EMT step has been reported to upregulate wound healing and the invasive activity of cancer cells $(19,20)$. Therefore, the migratory effects of mitochondrial respiration inhibitors were determined in A549 cells using the wound-healing assay. Compared with in the control group, treatment with $1 \mu \mathrm{g} / \mathrm{ml}$ mitochondrial respiration inhibitors for $24 \mathrm{~h}$ significantly (1.5-fold) promoted the migration of A549 cells (Fig. 4A). In addition, the effects of oligomycin A and antimycin A were determined on the invasion of A549 cells using the invasion assay. After $24 \mathrm{~h}$ treatment with oligomycin $\mathrm{A}$ and antimycin $\mathrm{A}$, the invasion of A549 cells was increased 4.25- and 2.07-fold, respectively (Fig. 4B). The invasive effects of oligomycin A were much higher than those of antimycin A. These results suggested that mitochondrial respiration inhibitors may enhance migration and invasion via induction of EMT in A549 cells.

Inhibitors of mitochondrial respiration mediate EMT through activation of the AKT and AMPK pathways in A549 cells. It has previously been reported that EMT is induced by factors, such as ROS, insulin receptor substrate-1 and hypoxia (21-23). Mitochondrial dysfunction caused by mitochondrial respiration inhibitors promotes cell migration through the induction of intracellular ROS in SC-M1 cells (14). Therefore, the present study aimed to determine whether oligomycin A and antimycin A may induce EMT through ROS. As presented in Fig. 5A and B, the ROS scavenger NAC had no effect on oligomycin A/antimycin A-reduced E-cadherin and oligomycin A/antimycin A-increased Vimentin.
Since previous studies indicated that the AMPK and AKT pathways control colony formation and cell proliferation, migration and invasion in several types of cancer (24-26), the present study investigated whether inhibitors of mitochondrial respiration may regulate the activation of AMPK, its downstream signaling substrate ACC, and AKT in A549 cells (Fig. 6). As shown in Fig. 6A, B, D, and E, oligomycin A and antimycin A significantly increased the expression levels of p-AMPK, p-ACC and p-AKT. In H1793 and H23 cells, p-AMPK and AKT expression levels were was significantly increased following treatment with $2 \mu \mathrm{g} / \mathrm{ml}$ oligomycin A and antimycin A (Fig. 6C and F). Therefore, in order to determine whether the two pathways are associated with oligomycin A/antimycin A-induced EMT, the effects of inhibitors of AMPK (Compound C, $10 \mu \mathrm{M}$ ) and AKT (wortmannin, $200 \mu \mathrm{M}$ ) were determined on EMT-associated proteins. Compound $\mathrm{C}$ suppressed oligomycin A/antimycin A-induced E-cadherin downregulation and oligomycin A/antimycin A-induced Vimentin upregulation, along with p-AMPK expression (Fig. 6G). Wortmannin also inhibited oligomycin A/antimycin A-regulated EMT marker protein expression (Fig. 6H). These results suggested that the AMPK and AKT signaling pathways may serve important roles in mitochondrial respiration inhibitors-induced EMT in lung cancer cells.

\section{Discussion}

Defective mitochondria were proposed by Otto Warbug to explain why tumor cells undergo increased aerobic glycolysis, namely the Warbug effect, unlike normal cells (27). Various mechanisms underlying dysregulated energy generation in cancer cells are associated with mitochondrial 
A

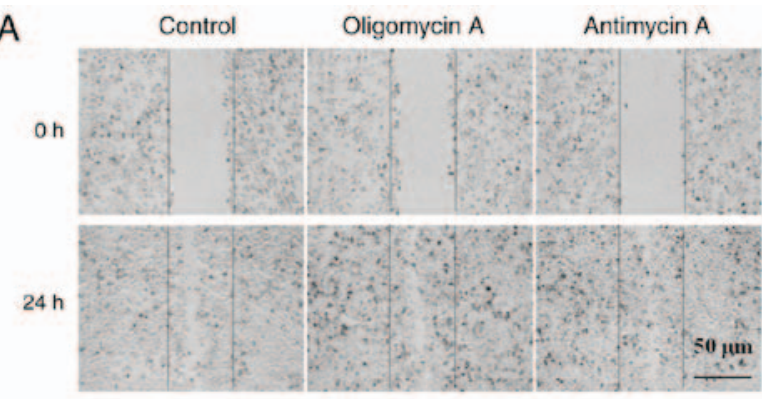

B

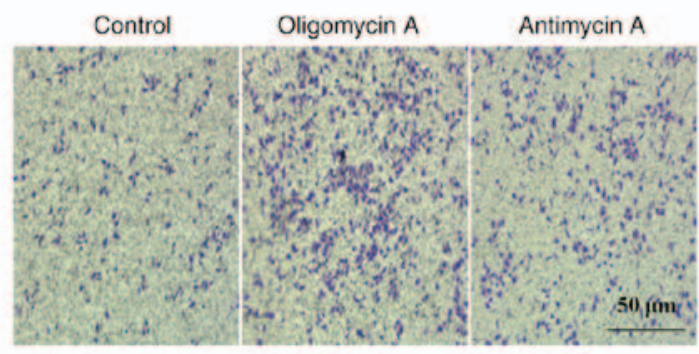

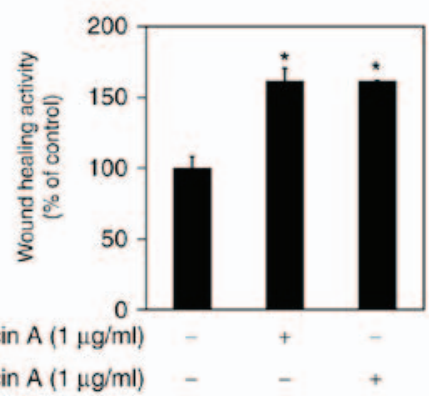

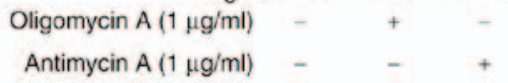

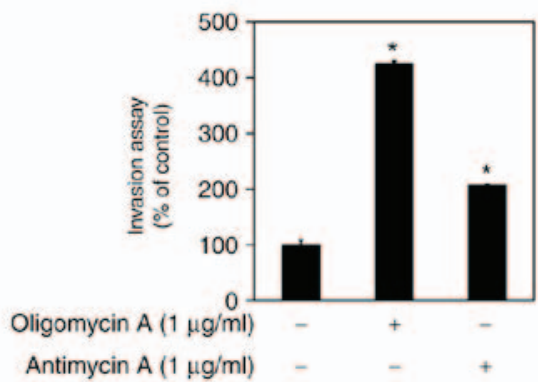

Figure 4. Inhibitors of mitochondrial respiration increase cell migration and invasion of A549 cells. (A) A scratch wound was made on the cell surface, and cells were treated with $1 \mu \mathrm{g} / \mathrm{ml}$ oligomycin A and antimycin A for $24 \mathrm{~h}$. Images of cells that migrated into the wound were captured (magnification, $\mathrm{x} 400$ ). (B) A549 cells were treated with $1 \mu \mathrm{g} / \mathrm{ml}$ oligomycin A and antimycin A for $24 \mathrm{~h}$ and were examined by a Transwell assay. Images of cells that invaded across the membrane were captured (magnification, $x 400$ ). The wound-healing and invasive activities of cells were determined as the number of each condition's cells divided by the number of control cells. Data are presented as the means \pm standard error of three independent experiments. ${ }^{*}<<0.05$ vs. the control group.

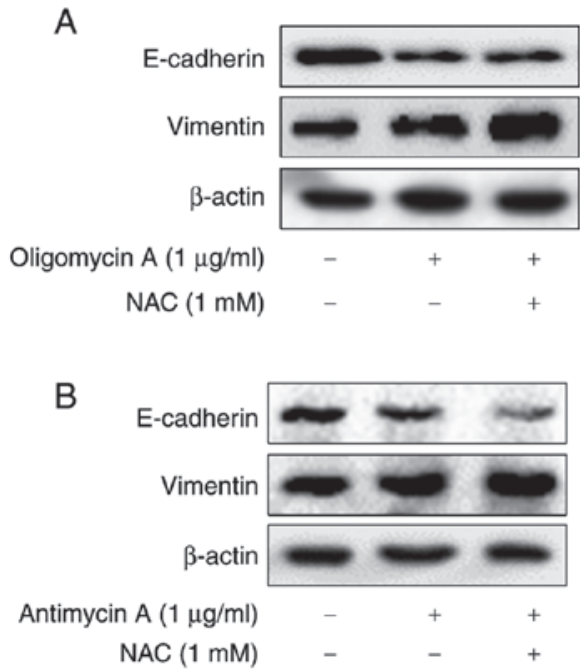

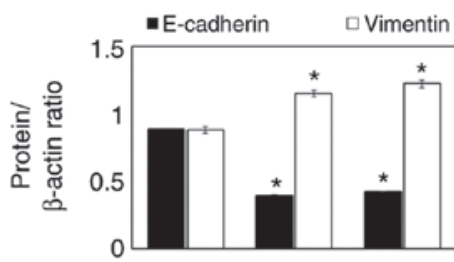

Oligomycin A $(1 \mu \mathrm{g} / \mathrm{ml}) \quad-\quad+\quad+$

$\mathrm{NAC}(1 \mathrm{mM}) \quad-\quad-\quad+$

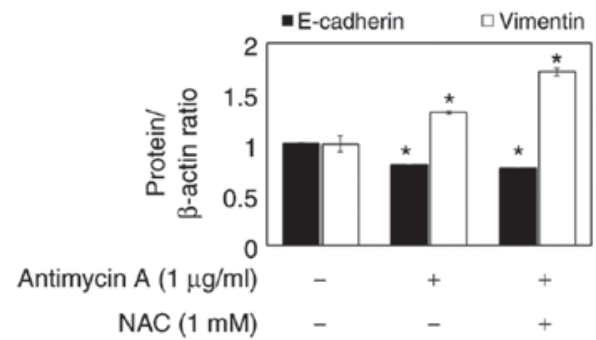

Figure 5. Effects of mitochondrial respiration inhibitors on EMT are not associated with reactive oxygen species in A549 cells. (A and B) A549 cells were incubated with $1 \mathrm{mM} \mathrm{NAC}$ in the absence or presence of $1 \mu \mathrm{g} / \mathrm{ml}$ oligomycin A or $1 \mu \mathrm{g} / \mathrm{ml}$ antimycin A for 24 h. Cell lysates were subjected to western blot analysis, to detect E-cadherin, Vimentin and $\beta$-actin protein expression. Densitometric analysis of each protein ratio was performed using ImageJ. Data are presented as the means \pm standard error of three independent experiments. ${ }^{*} \mathrm{P}<0.05$ vs. the control group. NAC, N-acetyl-L-cysteine.

dysfunction induced by mtDNA mutations, mutations in nuclear gene-encoded electron transport chain proteins and treatment with inhibitors of mitochondrial electron transport chains, including rotenone, oligomycin A and antimycin A (28-30). Previous studies have also suggested that impaired mitochondria may serve critical roles in tumor initiation and progression (1,31). For example, OXPHOS dysfunction has been reported to be involved in cell migration, invasion and metastasis (32). In addition, it was recently demonstrated that mitochondrial dysfunction promotes EMT through the transforming growth factor (TGF)- $\beta / \mathrm{Smad} / \mathrm{Snail}$ signaling pathway by activating c-Jun/activator protein-1 in hepatocellular carcinoma cells (33). Another study also demonstrated that mitochondrial dysfunction following TGF $\beta$-1 exposure induces acquisition of a mesenchymal morphology and the transfer from an epithelial to a mesenchymal phenotype in pancreatic cancer (28). The regulation of mitochondrial function by inhibitors of the mitochondrial electron transport chain, and their effects on EMT, cell migration and cell invasion, is critical but remains poorly understood in lung cancer research.

EMT is a pathophysiological process that is essential for the development of cancer cells, during which epithelial cells lose 

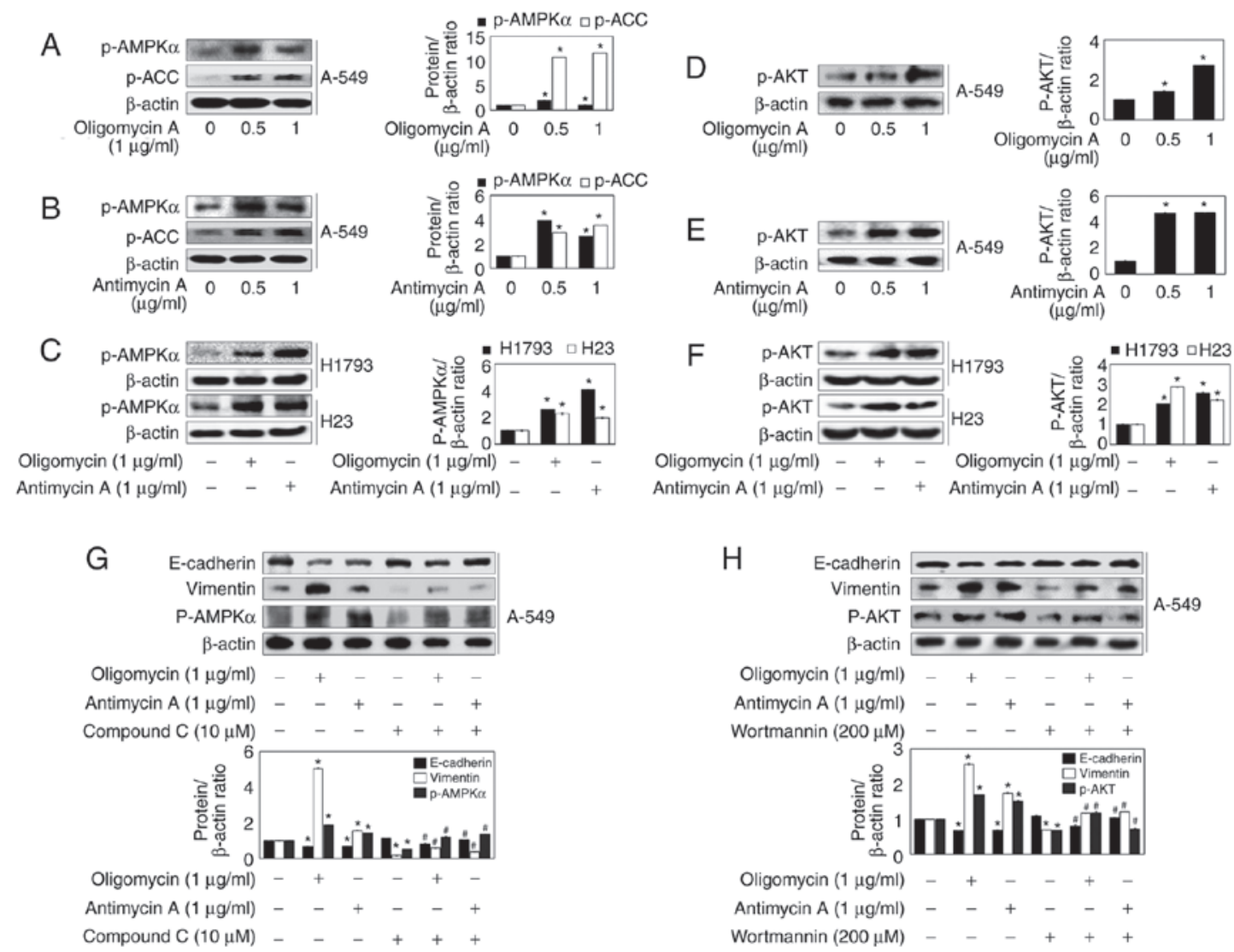

Oligomycin $(1 \mu \mathrm{g} / \mathrm{ml})$

Antimycin A $(1 \mu \mathrm{g} / \mathrm{ml})$

Wortmannin $(200 \mu \mathrm{M}$

Figure 6. Inhibitors of mitochondrial respiration modulate EMT through activation of AKT and AMPK signaling in A549 cells. (A-F) A549, H1793 and H23 cells were treated with the indicated concentrations of oligomycin A and antimycin A for $24 \mathrm{~h}$, and western blot analysis was conducted with antibodies against p-AMPK $\alpha$, p-ACC, p-AKT and $\beta$-actin. (G and H) A549 cells were treated with $1 \mu \mathrm{g} / \mathrm{ml}$ oligomycin A or $1 \mu \mathrm{g} / \mathrm{ml}$ antimycin A in the absence or presence of $10 \mu \mathrm{M}$ Compound $\mathrm{C}$ or $200 \mu \mathrm{M}$ wortmannin for $24 \mathrm{~h}$. The expression levels of E-cadherin, Vimentin, p-AMPK $\alpha$ and p-AKT were analyzed by western blot analysis. $\beta$-actin was used as a control. Densitometric analysis of each protein ratio was performed using ImageJ. Data are presented as the means \pm standard error of three independent experiments. ${ }^{*} \mathrm{P}<0.05$ vs. the control group, ${ }^{\prime} \mathrm{P}<0.05$ vs. the oligomycin A or antimycin A groups. ACC, acetyl-CoA carboxylase; AKT, protein kinase; AMPK, AMP-activated protein kinase; p-, phosphorylated.

their polarity and cell-cell adhesion is disrupted, thus resulting in transformation into mesenchymal cells through the reorganization of the cytoskeleton $(34,35)$. In the present study, the effects of two mitochondrial respiration inhibitors, oligomycin A and antimycin A, on phenotypic alterations associated with EMT were determined in H1793, H23 and A549 lung cancer cells. Oligomycin A and antimycin A have previously been reported to increase cell migration and expression of the mesenchymal protein marker Vimentin in SC-M1 human gastric cancer cells (14). The damaged function of mitochondrial OXPHOS has been demonstrated to initiate cell migration, invasion and metastatic properties by regulating the extracellular matrix $(36,37)$. As expected, the present study confirmed that oligomycin A and antimycin A increased EMT, and induced the transformation of cells into an invasive phenotype, thus suggesting that mitochondrial respiration inhibition may be associated with the invasion and migration of cancer cells.

During EMT, E-cadherin, which is an epithelial cell marker that is located on the basolateral membrane of adherens junctions, has been reported to be decreased due to the increase of mesenchymal protein markers, including Vimentin and $\mathrm{N}$-cadherin, and of typical transcription factors, including Snail, Slug, TWIST and zinc finger E-box-binding homeobox family proteins $(38,39)$. In addition, increased expression of Snail and Slug mRNA has a major role in EMT promotion in bladder cancer compared with in normal cells and tissues (40). In the present study, treatment with oligomycin A and antimycin A enhanced EMT by increasing the expression of Vimentin and inhibiting the expression of E-cadherin in $\mathrm{H} 1793$, H23 and A549 lung cancer cells. In addition, the protein and mRNA expression levels of Snail and Slug were increased by oligomycin A and antimycin A in A549 cells. These results indicated that mitochondrial respiration inhibitors may promote regulation of Vimentin and E-cadherin via induction of the transcription factors Snail and Slug.

The present study also investigated the molecular mechanisms through which oligomycin A and antimycin A operate as oncogenic agents in lung cancer. AMPK is a key regulator involved in maintaining energy homeostasis and regulating other physiological functions in cells (41). Activation of AMPK is promoted by various cellular environmental factors, including energy deficiency, hypoxia and oxidative stress caused by non-functional mitochondria (42). The present study revealed that oligomycin A and antimycin A upregulated the expression levels of p-AMPK. In addition, mitochondrial respiration inhibitor-induced E-cadherin downregulation and Vimentin upregulation were suppressed by Compound C (AMPK inhibitor) in A549 cells. Numerous studies have reported that ROS arising from mitochondrial dysfunction mediate EMT and cell invasion in various cancer cells via activation of the AMPK 
signaling pathway $(29,42)$. Furthermore, mitochondrial malfunction increases cell migration and invasion by increasing the production of ROS in SKBR3 breast cancer cells (1). However, in the present study, oligomycin A/antimycin A-regulated EMT marker protein expression was not affected by treatment with a ROS scavenger in A549 cells. This discrepancy between the present and previous studies may be due to the different cell types used. These results suggested that oligomycin A and antimycin A triggered EMT by activating AMPK independent of ROS in A549 cells.

The AKT pathway is known to serve an important role in intracellular processes, including glucose metabolism, cell proliferation and metastasis. It is also highly activated in various tumor cells (43). The mitochondrial stress induced by mitochondrial respiration inhibitors, including rotenone and oligomycin A, upregulates matrix metalloproteinase-13 expression and invasion by activating AKT pathway signaling in various cancer cells (44). In the present study, oligomycin A and antimycin A upregulated the expression levels of p-AKT in three types of lung cancer cells. In addition, inhibition of AKT phosphorylation suppressed oligomycin A- and antimycin A-induced E-cadherin downregulation and Vimentin upregulation, thus suggesting that inhibitors of mitochondrial respiration may initiate EMT by activating AKT pathway signaling in A549 cells.

In conclusion, the present study demonstrated that oligomycin A and antimycin A may induce mesenchymal-like morphological features, and cell migration and invasion, in various human lung cancer cell lines. Oligomycin A and antimycin A downregulated expression of the epithelial marker protein E-cadherin, and upregulated expression of the mesenchymal marker proteins Vimentin, Snail and Slug. In addition, oligomycin A and antimycin A induced the protein expression levels of mesenchymal markers via activation of the AKT and AMPK signaling pathways. These results indicated that inhibition of mitochondrial respiration may activate metastasis via EMT in lung cancer. Therefore, control of mitochondrial respiration in patients with lung cancer may be considered a novel approach for the prevention of metastasis.

\section{Acknowledgements}

Not applicable.

\section{Funding}

This study was supported by the KIST intramural research grant (grant no. 2Z04930) and also by the Basic Science Research Program through the National Research Foundation of Korea (NRF) funded by the Ministry of Education (grant no. NRF-2015R1D1A4A01019102).

\section{Availability of data and materials}

The datasets used and/or analyzed during the current study are available from the corresponding author on reasonable request.

\section{Authors' contributions}

YJJ and YCC designed the experiments. SYH and YJJ performed the experiments. YC, SKH and YSB analyzed the data, and contributed materials and analytical tools. SYH, YJJ and YCC wrote the manuscript. All authors read and approved the final manuscript.

\section{Ethics approval and consent to participate}

Not applicable.

\section{Patient consent for publication}

Not applicable.

\section{Competing interests}

The authors declare that they have no competing interests.

\section{References}

1. Ma J, Zhang Q, Chen S, Fang B, Yang Q, Chen C, Miele L, Sarkar FH, Xia J and Wang Z: Mitochondrial dysfunction promotes breast cancer cell migration and invasion through HIF1 $\alpha$ accumulation via increased production of reactive oxygen species. PLoS One 8: e69485, 2013.

2. Thiery JP: Epithelial-mesenchymal transitions in tumour progression. Nat Rev Cancer 2: 442-454, 2002.

3. Tsai JH and Yang J: Epithelial-mesenchymal plasticity in carcinoma metastasis. Genes Dev 27: 2192-2206, 2013.

4. Thiery JP, Acloque H, Huang RY and Nieto MA: Epithelial-mesenchymal transitions in development and disease. Cell 139: 871-890, 2009.

5. Ji Q, Liu X, Han Z, Zhou L, Sui H, Yan L, Jiang H, Ren J, Cai J and Li Q: Resveratrol suppresses epithelial-to-mesenchymal transition in colorectal cancer through TGF- $\beta 1 /$ Smads signaling pathway mediated Snail/E-cadherin expression. BMC Cancer 15: 97, 2015.

6. Hou P, Zhao Y, Li Z, Yao R, Ma M, Gao Y, Zhao L, Zhang Y, Huang B and Lu J: LincRNA-ROR induces epithelial-to-mesenchymal transition and contributes to breast cancer tumorigenesis and metastasis. Cell Death Dis 5: e1287, 2014.

7. Saxena M, Stephens MA, Pathak $H$ and Rangarajan A: Transcription factors that mediate epithelial-mesenchymal transition lead to multidrug resistance by upregulating $\mathrm{ABC}$ transporters. Cell Death Dis 2: e179, 2011.

8. Dumitriu IE, Dunbar DR, Howie SE, Sethi T and Gregory CD: Human dendritic cells produce TGF-beta 1 under the influence of lung carcinoma cells and prime the differentiation of CD $4^{+} \mathrm{CD} 25^{+} \mathrm{Foxp}^{+}{ }^{+}$regulatory T cells. J Immunol 182: 2795-2807, 2009.

9. Godlewski J, Nowicki MO, Bronisz A, Nuovo G, Palatini J, De Lay M, Van Brocklyn J, Ostrowski MC, Chiocca EA and Lawler SE: MicroRNA-451 regulates LKB1/AMPK signaling and allows adaptation to metabolic stress in glioma cells. Mol Cell 37: 620-632, 2010.

10. Godlewski J, Newton HB, Chiocca EA and Lawler SE: MicroRNAs and glioblastoma; the stem cell connection. Cell Death Differ 17: 221-228, 2010.

11. Kowaltowski AJ, de Souza-Pinto NC, Castilho RF and Vercesi AE: Mitochondria and reactive oxygen species. Free Radic Biol Med 47: 333-343, 2009.

12. Brandon M, Baldi P and Wallace DC: Mitochondrial mutations in cancer. Oncogene 25: 4647-4662, 2006.

13. Zamzami N, Susin SA, Marchetti P, Hirsch T, Gómez-Monterrey I, Castedo M and Kroemer G: Mitochondrial control of nuclear apoptosis. J Exp Med 183: 1533-1544, 1996.

14. Hung WY, Huang KH, Wu CW, Chi CW, Kao HL, Li AF, Yin PH and Lee HC: Mitochondrial dysfunction promotes cell migration via reactive oxygen species-enhanced $\beta 5$-integrin expression in human gastric cancer SC-M1 cells. Biochim Biophys Acta 1820: 1102-1110, 2012.

15. Chang CJ, Yin PH, Yang DM, Wang CH, Hung WY, Chi CW, Wei YH and Lee HC: Mitochondrial dysfunction-induced amphiregulin upregulation mediates chemo-resistance and cell migration in HepG2 cells. Cell Mol Life Sci 66: 1755-1765, 2009. 
16. Jeong JH, Kang SS, Park KK, Chang HW, Magae J and Chang YC: p53-independent induction of G1 arrest and p21WAF1/CIP1 expression by ascofuranone, an isoprenoid antibiotic, through downregulation of c-Myc. Mol Cancer Ther 9: 2102-2113, 2010.

17. Lin CW, Hou WC, Shen SC, Juan SH, Ko CH, Wang LM and Chen YC: Quercetin inhibition of tumor invasion via suppressing PKC delta/ERK/AP-1-dependent matrix metalloproteinase- 9 activation in breast carcinoma cells. Carcinogenesis 29: $1807-1815,2008$

18. Yi BR, Kim TH, Kim YS and Choi KC: Alteration of epithelial-mesenchymal transition markers in human normal ovaries and neoplastic ovarian cancers. Int J Oncol 46: 272-280, 2015.

19. Cano A, Perez-Moreno MA, Rodrigo I, Locascio A, Blanco MJ, del Barrio MG, Portillo F and Nieto MA: The transcription factor snail controls epithelial-mesenchymal transitions by repressing E-cadherin expression. Nat Cell Biol 2: 76-83, 2000.

20. Blanco MJ, Barrallo-Gimeno A, Acloque H, Reyes AE, Tada M, Allende ML, Mayor R and Nieto MA: Snailla and Snaillb cooperate in the anterior migration of the axial mesendoderm in the zebrafish embryo. Development 134: 4073-4081, 2007.

21. Hu Y, He K, Wang D, Yuan X, Liu Y, Ji H and Song J: TMEPAI regulates EMT in lung cancer cells by modulating the ROS and IRS-1 signaling pathways. Carcinogenesis 34: 1764-1772, 2013.

22. Lu X and Kang Y: Hypoxia and hypoxia-inducible factors: Master regulators of metastasis. Clin Cancer Res 16: 5928-5935, 2010.

23. Giannoni E, Parri M and Chiarugi P: EMT and oxidative stress: A bidirectional interplay affecting tumor malignancy. Antioxid Redox Signal 16: 1248-1263, 2012.

24. Han B, Cui H, Kang L, Zhang X, Jin Z, Lu L and Fan Z: Metformin inhibits thyroid cancer cell growth, migration, and EMT through the mTOR pathway. Tumour Biol 36: 6295-6304, 2015.

25. Gilmore TD: Introduction to NF-kappaB: Players, pathways, perspectives. Oncogene 25: 6680-6684, 2006.

26. Liu XL, Zhang XT, Meng J, Zhang HF, Zhao Y, Li C, Sun Y, Mei QB, Zhang $\mathrm{F}$ and Zhang T: ING5 knockdown enhances migration and invasion of lung cancer cells by inducing EMT via EGFR/PI3K/Akt and IL-6/STAT3 signaling pathways. Oncotarget 8: 54265-54276, 2017.

27. Upadhyay M,Samal J,Kandpal M,Singh OV and Vivekanandan P: The Warburg effect: Insights from the past decade. Pharmacol Ther 137: 318-330, 2013.

28. Guo Q: Changes in mitochondrial function during EMT induced by TGF $\beta-1$ in pancreatic cancer. Oncol Lett 13: 1575-1580, 2017.

29. Favre C, Zhdanov A, Leahy M, Papkovsky D and O'Connor R Mitochondrial pyrimidine nucleotide carrier (PNC1) regulates mitochondrial biogenesis and the invasive phenotype of cancer cells. Oncogene 29: 3964-3976, 2010.

30. Sequeira A, Martin MV, Rollins B, Moon EA, Bunney WE, Macciardi F, Lupoli S, Smith EN, Kelsoe J, Magnan CN, et al: Mitochondrial mutations and polymorphisms in psychiatric disorders. Front Genet 3: 103, 2012.
31. Kwong JQ, Henning MS, Starkov AA and Manfredi G: The mitochondrial respiratory chain is a modulator of apoptosis. J Cell Biol 179: 1163-1177, 2007.

32. Nunes JB, Peixoto J, Soares P, Maximo V, Carvalho S, Pinho SS, Vieira AF, Paredes J, Rego AC, Ferreira IL, et al: OXPHOS dysfunction regulates integrin- $\beta 1$ modifications and enhances cell motility and migration. Hum Mol Genet 24: 1977-1990, 2015.

33. Yi EY, Park SY, Jung SY, Jang WJ and Kim YJ: Mitochondrial dysfunction induces EMT through the TGF- $\beta / \mathrm{Smad} /$ Snail signaling pathway in Hep3B hepatocellular carcinoma cells. Int J Oncol 47: 1845-1853, 2015.

34. Pirozzi G, Tirino V, Camerlingo R, Franco R, La Rocca A, Liguori E, Martucci N, Paino F, Normanno N and Rocco G: Epithelial to mesenchymal transition by TGF $\beta-1$ induction increases stemness characteristics in primary non small cell lung cancer cell line. PLoS One 6: e21548, 2011.

35. Bao B, Wang Z, Ali S, Ahmad A, Azmi AS, Sarkar SH, Banerjee S, Kong D, Li Y, Thakur S and Sarkar FH: Metformin inhibits cell proliferation, migration and invasion by attenuating CSC function mediated by deregulating miRNAs in pancreatic cancer cells. Cancer Prev Res (Phila) 5: 355-364, 2012.

36. He X, Zhou A, Lu H, Chen Y, Huang G, Yue X, Zhao P and $\mathrm{Wu}$ Y: Suppression of mitochondrial complex I influences cell metastatic properties. PLoS One 8: e61677, 2013.

37. Imanishi H, Hattori K, Wada R, Ishikawa K, Fukuda S, Takenaga K, Nakada K and Hayashi J: Mitochondrial DNA mutations regulate metastasis of human breast cancer cells. PLoS One 6: e23401, 2011

38. Perez-Moreno M, Jamora C and Fuchs E: Sticky business: Orchestrating cellular signals at adherens junctions. Cell 112: $535-548,2003$.

39. Son $\mathrm{H}$ and Moon A: Epithelial-mesenchymal transition and cell invasion. Toxicol Res 26: 245-252, 2010.

40. Xu M, Li J, Wang X, Meng S, Shen J, Wang S, Xu X, Xie B, Liu B and Xie L: MiR-22 suppresses epithelial-mesenchymal transition in bladder cancer by inhibiting Snail and MAPK1/Slug/vimentin feedback loop. Cell Death Dis 9: 209, 2018.

41. Marshall S: Role of insulin, adipocyte hormones, and nutrient-sensing pathways in regulating fuel metabolism and energy homeostasis: A nutritional perspective of diabetes, obesity, and cancer. Sci STKE 2006: re7, 2006.

42. He K, Guo X, Liu Y, Li J, Hu Y, Wang D and Song J: TUFM downregulation induces epithelial-mesenchymal transition and invasion in lung cancer cells via a mechanism involving AMPK-GSK3 $\beta$ signaling. Cell Mol Life Sci 73: 2105-2121, 2016.

43. Manning BD and Cantley LC: AKT/PKB signaling: Navigating downstream. Cell 129: 1261-1274, 2007.

44. Li CH, Cheng YW, Liao PL, Yang YT and Kang JJ: Chloramphenicol causes mitochondrial stress, decreases ATP biosynthesis, induces matrix metalloproteinase-13 expression, and solid-tumor cell invasion. Toxicol Sci 116: 140-150, 2010. 